\title{
On the Possibility about Performance Estimation Just before Beginning a Voluntary Motion Using Movement Related Cortical Potential
}

\author{
Satoshi Suzuki ${ }^{1}$, Takemi Matsui ${ }^{1}$, Yusuke Sakaguchi ${ }^{1}$, Kazuhiro Ando ${ }^{1}$, \\ Nobuyuki Nishiuchi ${ }^{1}$, Toshimasa Yamazaki ${ }^{2}$, and Shin'ichi Fukuzumi ${ }^{3}$ \\ ${ }^{1}$ Tokyo Metropolitan University, Asahigaoka 6-6, Hino, Tokyo 191-0065, Japan \\ ${ }^{2}$ Kyusyu Institute of Technology, Kawazu 680-4, Iizuka, Fukuoka 820-8502, Japan \\ ${ }^{3}$ NEC Common Platform Software Research Laboratories, Shibaura 2-11-5, Minatoku, \\ Tokyo 108-8557, Japan \\ ssuzuki@cc.tmit.ac.jp
}

\begin{abstract}
The present study aimed to investigate this tripartite relationship, regarding MRCP as a physiological index, ballistic movement as an index of operation and accuracy of the task performance. Experiments were conducted 'reaching' task; the subject touches the target appears 300 pixels away from the start point in a vertical direction on the touch sensitive screen with the forefinger. During experiments, EEG, EMG as trigger, image by high-speed camera and the efficiency of task were acquired. As a result, significant differences between the high and poor performance groups were clear on the NS in MRCP acquired from $\mathrm{Fz}(p<0.05), \mathrm{Cz}(p<0.05)$ and $\mathrm{Pz}(p<0.05)$. Furthermore, the difference was confirmed on the duration of ballistic movement. Based on our findings, we attempted to extract MRCP rapidly and automatically without using signal averaging and discuss whether it is possible to estimate accuracy just before the motion is executed.
\end{abstract}

Keywords: Accuracy, ballistic movement, movement-related cortical potential (MRCP), reaching, voluntary motion.

\section{Introduction}

Movement-related cortical potential (MRCP) is a feature of brain waves that appears toward the midline of the head approximately one to two seconds before the onset of voluntary motion $[1,2,3]$. Although the divisions differ slightly between researchers, MRCP is usually separated into the Bereitschafts Potential (BP), the Intermediate Slope (IS) and the Negative Slope (NS). The BP and IS appear at the front and top of the head and have a gradual slope from $2000-500 \mathrm{msec}$ before movement, while the NS starts approximately $500 \mathrm{msec}$ before motion and, in the case of hand movement, has an amplitude of $10-15 \mu \mathrm{V}$ [3]. It can also be observed if the subject imagines the motion in their head without undertaking any real activity [4]. Recently, some studies have demonstrated a relationship between MRCP and eye movements [5], and 
to use as a method of medical diagnostics [6], although not all details have been fully clarified. Previous work has also shown that the thinking process and information processing within the brain is broken into several steps. Many useful models of this information processing in the head are proposed [7, 8]. These models commonly have 4 steps: perception, cognition, motion planning and motion. Related to this model, MRCP is generally assumed to reflect a preparation or planning stage for beginning the motion [9].

On the other hand, "reaching", such voluntary goal-directed movement, is known to be one of the most important components of motion by human arms. Mathematical models considering jerk, torque and dynamics of the musculoskeletal system have been used to understand how the motion of reaching is planned and controlled in the brain $[10,11,12]$. Movement efficiency has also been studied and Fitts' Law proved that reaction time during reaching constantly changes according to the difficulty of the task $[13,14]$. During the motion process reaching has two characteristics, the ballistic and corrective movements $[15,16]$, which influence both the accuracy and duration time of the motion. Observation of these characteristics enables us to comprehend the efficiency of the task performance. There is believed to be a relationship between the ballistic movement and MRCP as the former is a feed-forward movement and MRCP reflects a planning stage for this motion. Building on this, a relationship has also been suggested between MRCP, the ballistic movement and the accuracy of motion (Figure 1). Considering the tripartite relationship among MRCP as a physiological index, ballistic movement as an index of operation and accuracy of the task performance has meaning for ergonomics field.

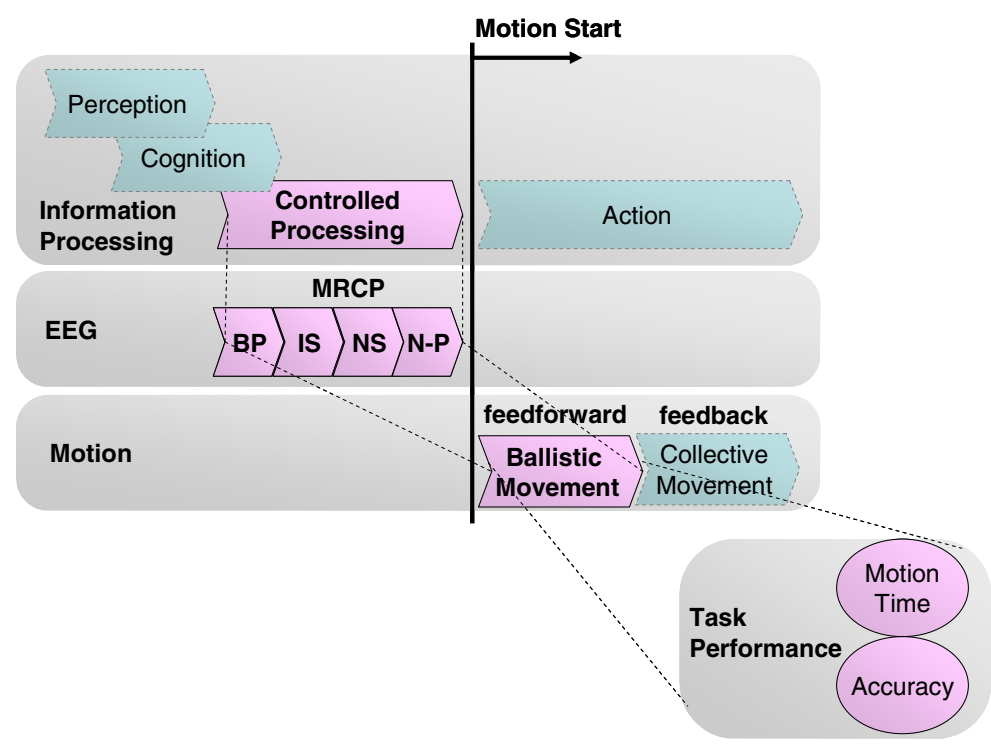

Fig. 1. The concept of this research. The aim of this study is an investigation the tripartite relationship, regarding MRCP as a physiological index, ballistic movement as an index of operation and accuracy of the task performance. 
The present study aimed to investigate this tripartite relationship, regarding MRCP as a physiological index, ballistic movement as an index of operation and accuracy of the task performance within the field of ergonomics. Based on our findings, we attempted to extract MRCP rapidly and automatically without using signal averaging. We explain and consider the results of this extracting system and discuss whether it is possible to estimate accuracy just before the motion is executed.

\section{Methods}

\subsection{Electroencephalogram}

MRCP is generally observed at scalp positions $\mathrm{Fz}, \mathrm{Pz}$ and $\mathrm{Cz}$, on the midline of the frontal and parietal regions of the head. To achieve high spatial resolution around these regions, electroencephalogram (EEG) data were acquired using a $128 \mathrm{ch}$ sensor net (Geodegic Sensor Net, Electrical Geodesics Inc., OR, USA) and analyze system (Net Station 4.3, Electrical Geodesics Inc., OR, USA). Electrode impedance was set between $10-50 \mathrm{k} \Omega$. EEG was recorded using a $0.1-50 \mathrm{~Hz}$ bandpass $(3 \mathrm{~dB}$ attenuation). Signals were sampled at $1 \mathrm{kHz}$ and were digitized.

\subsection{EMG and Trigger}

The trajectory of voluntary motion was observed using a high-speed camera (Fastcam512PCI, Fotron Co., Tokyo, Japan) with 125 fps (Figure 2). The trigger signal was generated using the surface EMG on the common digital extensor muscle. Data from the EEG and the high-speed camera were clipped at each trial using triggers. EMG data were acquired using a bio-amp system (Biotop, NEC-Sanei Co., Tokyo, Japan) with a sampling rate of $10 \mathrm{kHz}$ to accurately synchronize and analyze in real time using a Field Programmable Gate Array (FPGA) module (PCI-7831, National Instruments Co., TX, USA).

\subsection{Subjects and Task Procedure}

Experiments were conducted on eight healthy male subjects ranging in age from 22 to 24 years old (average $22.88+/-0.83$ years). All subjects were right-handed as confirmed by the Edinburgh handedness test [17]. Experiments were conducted in an electromagnetic-shielded room using the following protocols: the subject touches the center of a 17-inch touch-sensitive screen (LCD-AD172F2-T, I/O Data Co., Tokyo, Japan) located $30 \mathrm{~cm}$ in front of them with their forefinger. A small cross-shaped target then appears 300 pixels away from the point previously touched in a vertical direction (screen pixel pitch $0.264 \times 0.264 \mathrm{~mm}$ ). The subject moves their forefinger to the center of the displaying target and touches it. The trial is repeated a total of 50 times in two sets. Specific instructions regarding motion speed and accuracy during trial performance were not given to all subjects. The subject's head was placed on a jaw rest and the right forearm on an armrest to reduce artifacts other than motion of the forefinger. 


\subsection{Analysis}

MRCP and high-speed camera data were clipped in each trial by a trigger signal generated using the surface EMG on the common digital extensor muscle. EEG data were clipped from $1500 \mathrm{msec}$ before the start of the movement to $500 \mathrm{msec}$ afterward, according to previous studies.

The gap from the center of the target to the position actually touched was used as an evaluation index for the accuracy of the voluntary movement. Based on the gap average of task performance, we divided data of performance in each trial into two

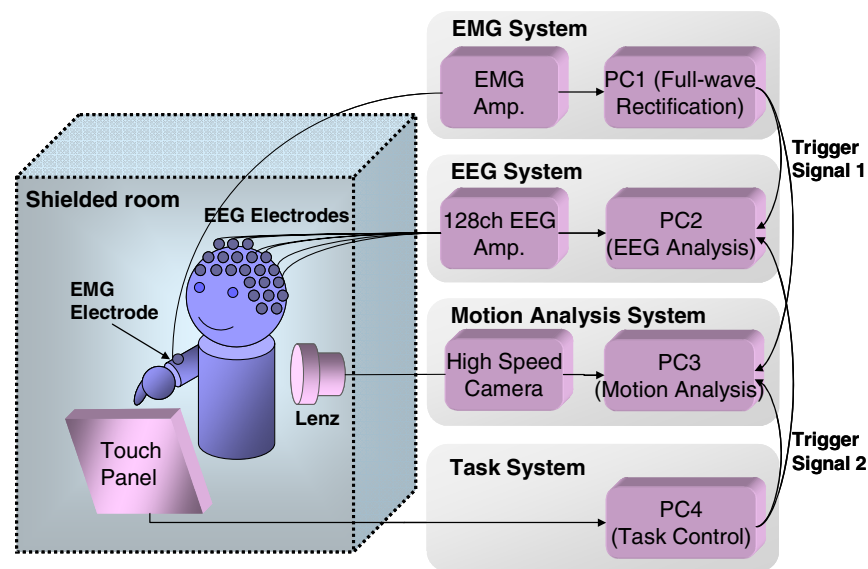

Fig. 2. Block diagram and data flow. Experiments were conducted on eight healthy male subjects using EEG, high-speed camera, EMG and task systems.

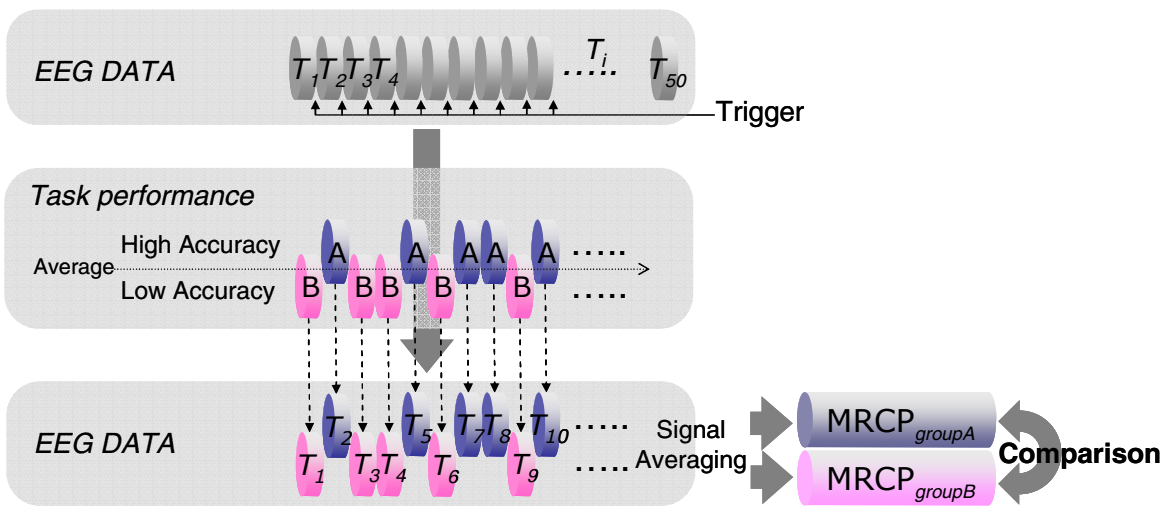

Fig. 3. Analytical process. MRCP and high-speed camera data were clipped in each trial by a trigger signal generated using the surface EMG. Based on the gap average of task performance, the clipped EEG and high-speed camera data from each trial were separated into two groups corresponding to the 'A' and 'B' groups. The typical waveforms in each group were calculated by averaging and compared with each other. 
groups: a high performance group 'A' and a low performance group 'B'. The clipped EEG and high-speed camera data from each trial were separated into two groups corresponding to the ' $\mathrm{A}$ ' and ' $\mathrm{B}$ ' groups. The typical waveforms in each group were calculated by averaging and compared with each other (Figure 3 ).

\section{Results}

\subsection{Task Performance}

Figure 4 shows the distribution of gaps in each trial according to a subject $\left(S_{I}\right)$. The distribution resembled a gamma distribution with an average gap of 5 pixels. The trend of the distribution form was seen in all subjects. EEG signals corresponding to trials when the gap was less than 5 pixels were placed in group A and those over 5 pixels were placed in group $\mathrm{B}$, in this subject $S_{I}$ 's case. Typical waveforms corresponding to the two groups were calculated by averaging. High-speed camera data were also divided into two groups.

\subsection{MRCP}

Figures 5 a) and Figure $5 \mathrm{~b}$ ) show sample of typical MRCP waveforms corresponding to the two groups acquired from $\mathrm{Fz}$ and $\mathrm{Cz}$ based on the same subject $S_{I}$ shown in Figure 4. Differences between BPs (observed from $2000 \mathrm{msec}$ before movement) and ISs (observed from 900 to 500 msec before movement) of groups A and B were not obvious. However, differences in NSs (observed from $500 \mathrm{msec}$ before movement) were clearly observed between the two groups, with group A showing a steeper slope than group $\mathrm{B}$. This trend was confirmed from around $\mathrm{Fz}, \mathrm{Cz}$, and $\mathrm{Pz}$ of all subjects (Figure 6) and the difference in average values between the two groups was found to be significant (Fz: $p<0.05(p=0.019)$, Cz: $p<0.05(p=0.050)$, Pz: $p<0.05(p=$ $0.017)$ ). This suggests that there is a relationship between the performance of the arm and the NS slope.

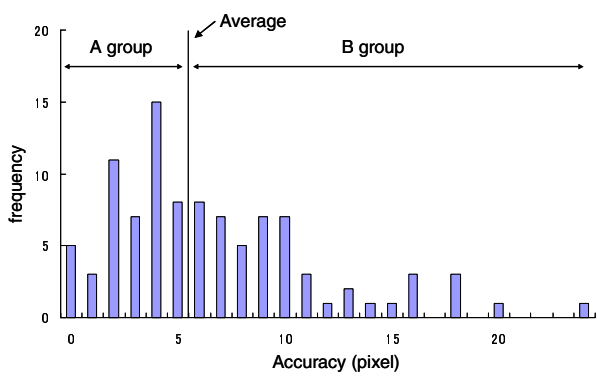

Fig. 4. Sample data of result of performance (Subject 1). The distribution resembled a gamma distribution with an average gap of 5 pixels, in this subject $S_{I}$ 's case. 


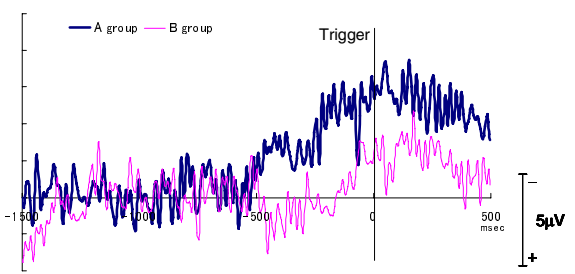

a) $\mathrm{Fz}$

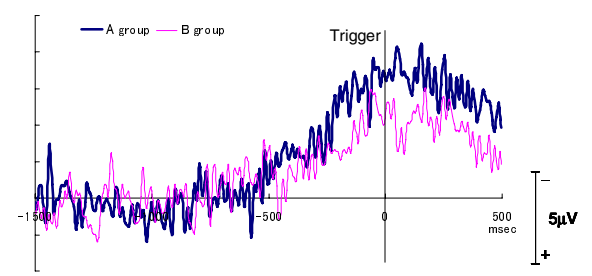

b) $\mathrm{Cz}$

Fig. 5. Sample data of MRCP (Subject 1). Typical MRCP waveforms corresponding to the two groups acquired from $\mathrm{Fz}$ (in Fig. 5 a)) and $\mathrm{Cz}$ (in Fig. 5 b))based on the same subject $S_{l}$ shown in Figure 4.

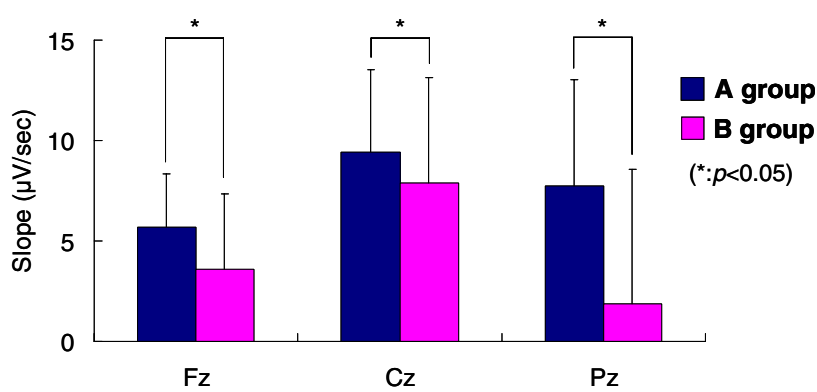

Fig. 6. Comparison of NS slopes in 2 groups (All subjects). Differences in NS (observed from $500 \mathrm{msec}$ before movement) were clearly observed between the two groups, with group A showing a steeper slope than group B.

\subsection{Process of the Reaching Motion}

Figure 7 shows the movement distance of the forefinger peak. A small difference in ballistic movement between groups A and B was confirmed, but we could not confirm a difference in corrective movement. This is because the experimental task is simple and primitive and the quantity allocated for corrective movement was few so it is not necessary to allocate for corrective movement.

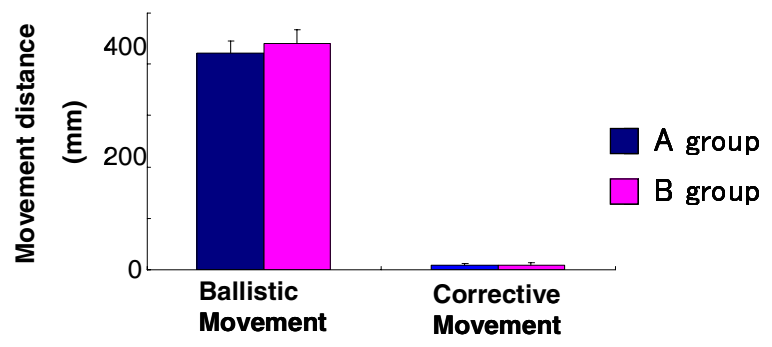

Fig. 7. Comparison of movement processes in 2 groups (All subjects). A small difference in ballistic movement between groups $\mathrm{A}$ and $\mathrm{B}$ was confirmed. 


\section{Discussion and Conclusion}

In the present study, we attempted to confirm the tripartite relationship between MRCP, ballistic movement and accuracy of task performance. We could not confirm any difference between high-performance group 'A' and low- performance group 'B' on BP and IS in MRCP acquired from $\mathrm{Cz}$ and Fz. However, significant differences between the groups were clear on the NS in MRCP acquired from $\mathrm{Cz}$ and Fz. Furthermore, the difference was confirmed on the duration of ballistic motion. As NS is generally believed to represent a preparation stage for voluntary motion, it appears that the observed difference between groups influences the process of motion and the task performance. These results show the possibility of estimating execution of the performance just before beginning the voluntary motion using MRCP.

On the other hand, the motion "reaching" is nearly optimized in terms of smoothness over the entire movement in the field considering with mathematical models. Various optimal criteria are proposed for trajectory planning of this motion in terms of multi-joint arm movements, like human arms. The minimum-jerk criterion [10] plans smooth trajectories in the extrinsic task space, while the minimum-joint-anglejerk criteria, the minimum-torque-change criteria [11], and the minimum-motorcommand-change criteria plan smooth trajectories [12] in the intrinsic body space. These models and criteria are discussed under the assumption that humans plan the trajectory of arm movement before beginning the motion. However, Hogan \& Wolpert [18] pointed out that it is difficult to explain the biological relevance of factors such as jerk or torque change in previous models of arm trajectory. They showed that the movement can be achieved by reducing the variance of errors at the end of the movement. The final goal of the "reaching" movement is to minimize the gap at the end of the motion, as suggested by their minimum-variance theory. Although it is not clear how the cerebrum and cerebellum contribute to achieving this movement, this model is an effective way of considering this movement. If we place our own results in context with this model, the concept becomes validated as it is known that humans plan and learn the trajectory of reaching with optimal efficiency just before beginning the motion.

Finally in the current study, we attempted to develop a prototype system to derive the NS slope in MRCP from EEG data automatically and in real time. When we observe the P300 and N200, the importance of the shape of the waveform necessitates signal averaging. If the event-related potentials (ERP), such as N200 and P300, was observed, the technique signal averaging is generally used not only to remove the artifact but also to extract more information, i.e. latency, amplitude, the shape of the waveform and so on. However, in the case of MRCP, we only need to observe slopes during the $500 \mathrm{~ms}$ just before the motion is executed, namely, just before the trigger. We are therefore attempting to develop a prototype system that obtains a sequentialmemory of the value during $2000 \mathrm{~ms}$ of EEG and sequentially calculates the value of the NSs slope in $500 \mathrm{~ms}$. This prototype system is currently being developed using LabVIEW (National Instruments Co., TX, USA) and we already confirmed that the performance can be estimate with $75 \%$ maximum of the time using an index based on NS values. Thus, in the future, our concept and method appears to be promising for use controlling devises safety, such as at car driving. 


\section{References}

1. Kornhuber, H.H., Deecke, L.: Hirnpotentialanderungen bei Willkurbewegungen und passiven Bewegungen des Menschen. Bereitschafts potential und reaffernte potentiale. Pfligers Arch, Gesamte Physiol Menschen Tiere 284, 1-17 (1965)

2. Barrett, G., Shibasaki, H., Neshige, R.A.: Computer-assisted method for averaging movement-related cortical potentials with respect to EMG onset. Electroencephalogr. Clin. Neurophysiol. 60(3), 276-281 (1985)

3. Shibasaki, H., Hallett, M.: What is the Bereitschaftspotential? Clin. Neurophysiol 117(11), 2341-2356 (2006)

4. MacKay, D.M., MacKay, V.: Behind the eye. Basil Blackwell, Malden (1991)

5. Yamamoto, J., Ikeda, A., Satow, T., et al.: Human eye fields in the frontal lobe as studied by epicortical recording of movement-related cortical potentials. Brain 127, 873-887 (2004)

6. Barrett, G., et al.: Cortical potential shifts preceding voluntary movements are nomal in parkinsonism. Electroencephalogr. Clin. Neurophysiol. 63, 340-348 (1986)

7. Card, S.K., Moran, T.P., Newell, A.: The psychology of human-computer interaction. Lawrence Erlbaum Associates, New Jersey (1983)

8. Gopher, D., Sanders, A.F.: S-Oh-R: Oh Stages! Oh Resources! In: Prinz, W., Sanders, A.F. (eds.) Cognition and motor processes, Springer, Heidelberg (1984)

9. Revelle, W.: Indivisual differences in personality and motivation: 'non-cognitive' determinants of cognitive performance. In: Baddeley, A., Weiskrantz, L. (eds.) Awareness Control, Clarendon Press, Oxford (1991)

10. Flash, T., Hogan, N.: The Coordination of Arm Movements: An Experimentally Confirmed Mathematical Model. Journal of Neuroscience 5, 1688-1703 (1985)

11. Uno, Y., Kawato, M., Suzuki, R.: Formation and control of optimal trajectory in human multijoint arm movement - Minimum torque-change model. Biological Cybernetics 61(2), 89-101 (1989)

12. Kawato, M.: Optimization and learning in neural networks for formation and control of coordinated movement. In: Meyer, D., Kornblum, S. (eds.) Attention and performance XIV, pp. 821-849. MIT Press, Cambridge (1993)

13. Fitts, P.M., Peterson, J.R.: Information capacity of discrete motor responses. Journal of Experimental Psychology 67, 103-112 (1964)

14. Accot, J., Zhai, S.: Beyond Fitts' Law: Models for trajectory-based HCI tasks. In: CHI 1997, pp. 295-302 (1997)

15. Flowers, K.: Ballistic and corrective movement on an aiming task. Neurology 25, 413-421 (1975)

16. Pratt, J., Abrams, R.A.: Practice and component submovements: The roles of programming and feedback in rapid aimed limb movements. Journal of Motor Behavior 28(2), 149-156 (1996)

17. Oldfield, R.C.: The assessent and analysis of handedness: The Edinburgh Inventory. Neuropsychologia 9, 97-113 (1971)

18. Harris, C.M., Wolpert, D.M.: Signal-dependent noise determinations motor planning. Nature 394, 780-784 (1998) 